\title{
Processo de trabalho e cuidado em saúde mental no Centro de Atenção Psicossocial da UERJ na pandemia de COVID-19
}

\author{
Anália da S. Barbosa, ${ }^{1 *}$ Clara V. Nascimento, ${ }^{1}$ Lucas B. S. Dias, ${ }_{1}^{1}$ Tiago B. do Espírito Santo, ${ }^{2}$ Renata da C. S. \\ Chaves, ${ }^{3}$ Tatiana C. Fernandes ${ }^{1}$
}

\section{Resumo}

Introdução: Trata-se de um relato do processo de trabalho e da produção de cuidados em saúde mental no Centro de Atenção Psicossocial II da Universidade do Estado do Rio de Janeiro durante o primeiro mês de pandemia de COVID-19. Desde 30 de janeiro de 2020, quando a COVID-19 foi declarada pela Organização Mundial da Saúde (OMS) como emergência de saúde pública de interesse internacional, os países vêm empreendendo esforços para organizar seus serviços de saúde para o atendimento com qualidade ao quantitativo de infectados e a crescente demanda por enfermarias e leitos de tratamento intensivo. Objetivos: relatar e analisar a reorganização dos processos de trabalho do CAPS UERJ, em virtude da política pública que determina o distanciamento social em meio à pandemia da COVID-19, considerando suas especificidades e seu compromisso com a lógica territorial. Materiais e Métodos: Pesquisa descritiva com caráter exploratório e abordagem qualitativa, do tipo relato de experiência. A coleta de dados se deu entre março e abril de 2020. Resultados: Ainda que não seja um serviço voltado ao atendimento dos acometidos pela COVID-19, o CAPS UERJ vem trabalhando na direção de assegurar a implementação do que foi preconizado pela OMS na organização e nos processos de trabalho que garantam a continuidade dos cuidados em saúde mental. Conclusões: A pandemia e seus desdobramentos no cenário socioeconômicoe cultural e mais diretamente nas trajetórias dos nossos usuários e seus familiares ressaltou a necessidade do trabalho no território, estreitando a comunicação com outros serviços de saúde e de outras políticas públicas.

Descritores: Pandemias; Coronavírus; Saúde mental;Serviços de saúde mental.

\section{Abstract \\ Work process and mental health care at the Psy- chosocial Care Center of UERJ in the COVID-19 pandemic}

Introduction: This is an experience report about the work process and the mental health care at the Psychosocial Care Center II of the State University of Rio de Janeiro (CAPS UERJ) during the first month of the COVID-19 pandemic. Since January 30, 2020, when COVID-19 was declared by the World Health Organization (WHO) as a public health emergency of international interest, countries have been making efforts to organize their health services to provide quality care to the number of infected and growing demand for intensive care. Objectives: To report and analyze the reorganization of CAPS
1. Centro de Atenção Psicossocial, Policlínica Piquet Carneiro, Universidade do Estado do Rio de Janeiro. Rio de Janeiro, RJ, Brasil.

2. Departamento de Enfermagem Médico Cirúrgica, Faculdade de Enfermagem, Universidade do Estado do Rio de Janeiro. Rio de Janeiro, RJ, Brasil.

3. Departamento de Alimentação e Nutrição, Policlínica Piquet Carneiro, Universidade do Estado do Rio de Janeiro, Rio de Janeiro, RJ, Brasil.

*Endereço para correspondência:

Avenida Marechal Rondon, 381

Rio de Janeiro, Brazil.

CEP 20950-003

E-mail: analia.socialrj@gmail.com

BJHBS, Rio de Janeiro, 2020;19(1):11-19

Received on 05/06/2020. Approved on 06/09/2020.

UERJ's work processes, in view of the public political virtue that determines social distance in the midst of the COVID-19 pandemic, considering its specificities and its commitment to territorial logic. Materials and Methods: Descriptive research with an exploratory character and qualitative approach, an experience report type. Data collection took place between March and April 2020. Results: Although it is not a service aimed at serving those affected by COVID-19, CAPS UERJ, has been working to ensure the implementation of what was recommended by WHO, in the organization and work processes that guarantee continuity of mental health care. Conclusions: The pandemic and its consequences in the socioeconomic and cultural scenario and more directly in the trajectories of our users and their families highlighted the need for work in the territory, strengthening communication with other health services and other public policies.

Keywords: Pandemics; Coronavirus; Mental mealth; Mental Health Services.

\section{Resumen}

Proceso de trabajo y atención de salud mental en el Centro de Atención Psicosocial de UERJ en la pandemia de COVID-19

Introducción: Esta es una descripción del proceso de trabajo y de la producción de atención de salud mental en el Centro de 
Atención Psicosocial II de la Universidade do Estado do Rio de Janeiro durante el primer mes de la pandemia de COVID-19. Desde el 30 de enero de 2020, cuando COVID-19 fue declarado por la Organización Mundial de la Salud (OMS) como una emergencia de salud pública de interés internacional, los países han hecho esfuerzos para organizar sus servicios de salud para brindar atención de calidad a la cantidad de personas infectadas y creciente demanda de salas y camas de cuidados intensivos. Objetivos: Informar y analizar la reorganización de los procesos de trabajo de CAPS UERJ, en vista de la virtud política pública que determina la distancia social en medio de la pandemia de COVID-19, considerando sus especificidades y su compromiso con la lógica territorial. Materiales y Métodos: Investigación descriptiva con carácter exploratorio y enfoque

\section{Introdução}

O presente estudo tem como objeto o relato do processo de trabalho e da produção de cuidados em saúde mental no Centro de Atenção Psicossocial II da Universidade do Estado do Rio de Janeiro (CAPS UERJ) durante o início de pandemia da COVID-19.

A COVID-19 foi declarada como uma emergência de saúde pública de interesse internacional, em 30 de janeiro de 2020, pelo Diretor-Geral da Organização Mundial da Saúde (OMS). A partir deste marco, em âmbito global, os países vêm empreendendo esforços para organizar seus serviços de saúde de modo a atender à crescente demanda por enfermarias e leitos de tratamento intensivo, ${ }^{1}$ tendo sido registrado, em 2 de abril de 2020, a marca de um milhão de infectados e taxa de mortalidade de 5,2\%, em 204 países e territórios. ${ }^{2}$

Nessa conjuntura, destaca-se a particularidade de grupos socialmente vulneráveis que estão mais expostos ao risco de contaminação por se encontrarem em condições mais precárias de vida. Acredita-se que em meio à pandemia da COVID-19, os grupos de risco não englobam apenas idosos, pessoas com comorbidades, pessoas em situação de rua, mas também pessoas de grupos socioeconômicos que possam ter dificuldades para lidar financeiramente, mentalmente ou fisicamente com a crise.

A pandemia sobrecarrega os sistemas de saúde, provocando um aumento no quantitativo de mortalidades diretamente relacionadas ao surto, mas também nos índices de mortalidade indireta, vinculadas às condições evitáveis e tratáveis. Análises acerca do surto de Ebola, entre 2014 e 2015, apontam que o quantitativo de mortes por malária, sarampo, HIV e tuberculose foi maior do que aqueles relacionados à epidemia propriamentedita.Desta forma, aponta-se para cualitativo, un tipo de informe de experiencia. La recopilación de datos ocurrió entre marzo y abril de 2020. Resultados: Aunque no es un servicio destinado a atender a los afectados por COVID-19, CAPS UERJ ha trabajado para garantizar la implementación de lo recomendado por la OMS, en los procesos de organización y trabajo que garantizan la continuidad de la atención de salud mental. Conclusiones: La pandemia y sus consecuencias en el escenario socioeconómico y cultural y más directamente en las trayectorias de nuestros usuarios y sus familias destacaron la necesidad de trabajar en el territorio, fortaleciendo la comunicación con otros servicios de salud y otras políticas públicas.

Palabras clave: Pandemias; Salud mental; Coronavirus; Servicios de Salud Mental. a necessidade de manutenção de um acesso equitativo à prestação de serviços essenciais durante uma emergência, reduzindo assim o número de mortalidade direta e indireta. ${ }^{3}$ Este dado ressalta a necessidade de uma organização que possibilite o funcionamento dos serviços essenciais à saúde, garantindo força de trabalho para lidar com necessidades para além da COVID-19. ${ }^{3}$

Dentre os serviços essenciais, a OMS destacou aqueles que garantem o calendário de vacinação, os cuidados durante a gravidez e parto, o tratamento de doenças infecciosas e não transmissíveis, os serviços de sangue e os dispositivos da rede de saúde mental, o que, na realidade brasileira, refere-se aos pontos da Rede de Atenção Psicossocial (RAPS) no âmbito do Sistema Único de Saúde (SUS). Destaca-se também a importância de medidas que assegurem o cuidado aos idosos, refugiados e outros grupos vulneráveis, garantindo o bem-estar das pessoas que perderam sua renda e precisam de serviços essenciais, trabalhando, junto à comunidade para assegurar o apoio à resiliência e à saúde mental. ${ }^{3}$

Em território nacional, segundo Ministério da Saúde (MS) o quantitativo insuficiente de exames impediu a testagem em massa da população, o que possibilitaria a identificação prévia dos infectados, favoreceria a busca ativa e priorizaria seu isolamento e cuidado. Para os suspeitos, o protocolo é o autoisolamento domiciliar, sem a necessidade de notificação às autoridades sanitárias, tendo como consequência disso a provável redução da magnitude da curva epidêmica de COVID-19 brasileira. Esta política pública de manejo de crise influencia diretamente o campo da saúde mental, uma vez que este é subsidiado pela Reforma Psiquiátrica Antimanicomial Brasileira (RPB) e os pressupostos da 
Reabilitação Psicossocial, da desinstitucionalização e do cuidado no território. ${ }^{45}$

A RPB reconduziu as práticas de saúde mental, marcando-as por uma condução teórico-éticometodológica que afirmou uma produção de cuidados que extrapola o campo biomédico, em direção a uma proposta de mudança societária por meio de ações comunitárias e territoriais voltados para o direito à moradia, ao trabalho e ao lazer dos usuários, reabilitação psicossocial, tendo o território como produtor de conexões que viabilizam a produção de vida dos sujeitos. Desta maneira, por potencializar a circulação na cidade, a produção de encontros, as ações territoriais são ferramentas de Reabilitação Psicossocial e da Desinstitucionalização que viabilizam redes de conexões existenciais para ampliação da potência de vida desses sujeitos. ${ }^{6}$

A partir da avaliação da vulnerabilidade e da necessidade da continuidade de acompanhamento em saúde mental no período de distanciamento social, tornou-se imperativa a questão norteadora: Como a equipe do CAPS UERJ, mobilizada pela conjuntura da pandemia da COVID-19, pode dar continuidade ao cuidado em saúde mental e ressignificar seu processo de trabalho na direção de produzir cuidados territoriais, não ambulatoriais, em virtude de uma política pública atual que preconiza distanciamento social?

Este artigo tem como objetivos, portanto, relatar e analisar a reorganização dos processos de trabalho do CAPS UERJ em virtude da política pública que determina distanciamento social devido à pandemia da COVID-19. Considera as especificidades e o compromisso desse trabalho com a lógica territorial advogada pela RPB a fim de que possa contribuir para a estruturação de outros dispositivos de atendimento de pessoas acometidas por transtornos mentais graves, severos e persistentes, na direção de preservar a lógica territorial.

\section{Materiais e métodos}

Trata-se de uma pesquisa descritiva com caráter exploratório e abordagem qualitativa, por compreendêla adequada à captação de uma realidade não quantificável do mundo dos significados das ações e das relações humanas, ampliando-se assim o conhecimento profissional e melhoria na qualidade do atendimento? Utilizou-se a estratégia do relato de experiência, cujo objetivo é captar uma realidade específica que, por sua dinâmica, demanda constantes atualizações. ${ }^{8}$

Os dados foram coletados de março a abril de 2020, período do processo de planejamento, implementação e ações de fluxo de atendimentos aos usuários inscritos no CAPS UERJ durante o período inicial da pandemia de COVID-19, no município do Rio de Janeiro.

O CAPS UERJ, fundado em 2009, localiza-se no complexo ambulatorial da Policlínica Piquet Carneiro (PPC), no Município do Rio de Janeiro, tendo sob sua responsabilidade a Área Programática (AP) 2.2, que abrange os bairros: Grajaú, Andaraí, Vila Isabel, Tijuca, Saens Peña, Maracanã, Praça da Bandeira, Alto da Boa Vista e Usina. Segundo o último censo, realizado em 2010, a população adstrita a esse território é de 371.120 habitantes. ${ }^{9}$ Tal localização do CAPS impõe o desafio de sustentar o funcionamento de um serviço de alta complexidade em saúde mental dentro de uma Policlínica com atendimento de caráter ambulatorial.

O CAPS UERJ está inserido em uma estrutura universitária e é municipalizado, integrando assim a rede do município de saúde mental do Rio de Janeiro. A equipe é composta por: psicólogos, enfermeiros, assistentes sociais, psiquiatras, terapeutas ocupacionais, nutricionistas, técnicos de enfermagem, técnicos administrativos e oficineiros. Dentre estes, estão residentes, estagiários, professores e profissionais servidores do Estado. Logo, a instituição se configura como um polo de formação da força de trabalho para a Saúde Mental.

Por ser um CAPS do tipo II, o serviço funciona com a lógica da "Porta Aberta", de 8 h às $17 \mathrm{~h}$, de segunda a sexta, tendo como perfil de atendimento portadores de transtornos mentais graves e persistentes proveniente de diversos dispositivos entre eles as doze Clínicas da Família do território, Hospitais Psiquiátricos e, também, por demanda espontânea. ${ }^{10}$ Atualmente, o CAPS UERJ possui 317 usuários cadastrados, 57 em processo de recepção, com uma frequência diária que oscila entre 60 a 80 atendimentos, com prevalência de indivíduos do sexo masculino, de 30 a 50 anos, e uma parcela significativa encontra-se em situação vulnerabilidade social e econômica.

Em consonância com as diretrizes e objetivos expostos na Portaria $\mathrm{n}^{0} 3.088,{ }^{11}$ que institui a Rede de Atenção Psicossocial, o processo de cuidado no CAPS UERJ prioriza o desenvolvimento de atividades no território favorecendo a inclusão social, o estabelecimento de ações intersetoriais para garantir a integralidade do cuidado e a realização de atividades em espaços coletivos (grupos, assembleias, oficinas, reunião semanal de equipe, reunião de começo e final de turno, fóruns). ${ }^{11}$ A centralidade dos espaços coletivos atravessa o cotidiano dos trabalhadores e o processo de 


\section{Artigo original}

cuidado dos usuários e seus familiares.

Atualmente, o dia no CAPS UERJ tem seu início e término com uma reunião dos profissionais para discussão e organização das atividades e após é realizado o "Bom dia", um grupo de acolhimento diário para usuários e seus familiares. A partir desse acolhimento iniciam-se no espaço interno do CAPS as atividades em grupo e oficinas, como ,por exemplo, a oficina de mosaico, bijuterias, simbolização e uma oficina que promove a circulação dos usuários pela cidade; os atendimentos individuais dos usuários e de seus familiares pela equipe multiprofissional; as assembleias e a livre circulação pelos espaços do CAPS que promovem encontros entre usuários, familiares e trabalhadores (convivência). Para além do espaço físico do CAPS, ocorrem as atividades no território, como o acompanhamento dos usuários e familiares em suas variadas demandas e necessidades e as visitas domiciliares e institucionais (serviços de Assistência Social, Educação, Justiça e Saúde). Assim, fica nítido que circulação, coletivo, encontros, convivência e espaço urbano são elementos cruciais para a operacionalização do trabalho.

A equipe de pesquisa foi composta por um grupo de seis trabalhadores do CAPS que utilizaram, como técnica de coleta de dados, Diários de Campo no qual foram registradas as experiências vivenciadas no cotidiano institucional, sobretudo no que tange à recriação dos fluxos institucionais e processos de trabalho. As anotações seguiram a organização em três partes: a descrição dos acontecimentos, a interpretação do observado e o registro de conclusões preliminares, tal como dúvidas e imprevistos. ${ }^{12}$

Para a confecção do Diário de Campo, cada pesquisador utilizou-se de registros diários gravados que foram posteriormente transcritos e unificados em um único documento textual que aglutinou todas as experiências. Foram excluídos relatos com informações ausentes, incompreensíveis, realizados fora do período estipulado ou não relacionadas ao objeto de estudo. Para a análise do material, foi utilizada a literatura de suporte na área temática em questão.

Este artigo atende às normativas de pesquisas da Resolução n. ${ }^{\circ}$ 510, de 7 de abril de 2016, do Conselho Nacional de Saúde, não sendo necessário o uso de Termo de Consentimento Livre e Esclarecido (TCLE) ou aprovação no Comitê de Ética e Pesquisa. ${ }^{13}$

\section{Resultados}

Reorganização dos fluxos e do processo de trabalho do CAPS UERJ frente à pandemia de coronavírus
A OMS, ao decretar pandemia por COVID-19, em 11 de março de 2020, estabeleceu orientações para a organização e gerenciamento dos centros de tratamentos específicos. Destacaram-se, dentre esses protocolos estabelecidos, três principais intervenções: triagem nas unidades de saúde; a criação de instalações comunitárias para o cuidado de pacientes leves e; centros de treinamento e direcionamento para enfermarias ou similares específicos para os casos mais graves. ${ }^{14}$ No âmbito das singularidades de um serviço de saúde mental, ressaltaram-se: coordenação, planejamento e monitoramento; comunicação de risco e envolvimento da comunidade; vigilância, equipes de resposta rápida, e investigação de caso; gestão de caso; e suporte de operações e logística. ${ }^{1}$ Dentre as recomendações básicas estão a indicação para o adiamento ou suspensão de serviços eletivos e rotineiros, fluxos efetivos de pacientes (com uma triagem prévia e direcionamento de casos de COVID-19) e visitas de rotina para a promoção de saúde. ${ }^{3}$

Diante da realidade apresentada, a equipe do CAPS UERJ implementou um Grupo de Trabalho (GT) composto por membros da gestão e trabalhadores, objetivando realizar discussões e deliberar ações voltadas à manutenção da produção de cuidados e ao aprimoramento da prevenção dos usuários e colaboradores. Para contemplar estes objetivos foram necessárias a reorganização do processo de trabalho e a reconfiguração dos fluxos de informações para a comunidade interna ao CAPS UERJ (funcionários, usuários e acompanhantes).

Ações de educação em saúde abordando temas sobre cuidados básicos de higiene para a prevenção do contágio ocorreram diariamente durante a atividade denominada "Bom Dia", enquanto o isolamento social ainda não era uma determinação do MS.

O debate de informações acerca da higiene é urgente ao considerar-se a particularidade dos casos acompanhados pelo serviço. Falamos aqui de indivíduos e famílias que, em muitos casos, não possuem o mínimo do acesso a questões básicas de subsistência e saneamento básico. Portanto, orientar essas famílias quanto o autocuidado possível diante das condições que se apresentavam, também foi um desafio.

A intervenção está alinhada às orientações da Superintendência de Saúde Mental (SSM) do Rio de Janeiro, que determinou em documento oficial, publicado em 16 de março, que as organizações da estrutura e rotinas internas dos serviços deveriam, além de atentar para a higienização dos CAPS e seus 
mobiliários, abordar ações educativas em saúde, voltadas à instrução da comunidade..$^{14}$

Com o aparecimento dos primeiros casos no Brasil de infecção por SARS-CoV-2, o MS deliberou novas recomendações na direção do isolamento e distanciamento social. Para tanto, o GT foi convocado para reuniões com o diretor interino da PPC e outros serviços para definir o funcionamento da unidade nessa conjuntura. Estas reuniões foram o marco inicial para a definição de novos fluxos de triagem para todos os frequentadores da Policlínica que, a partir deste momento, passariam por avaliação da equipe de enfermagem e médicos. A triagem dividiu os pacientes em três grupos: o primeiro com livre circulação (assintomáticos); o segundo, com sintomas brandos da COVID-19, sendo testados, encaminhados para distanciamento social e monitorados por telefone enquanto aguardam o resultado; e o terceiro grupo, com sintomatologia grave, necessitando de cuidados intensivos.

Diante da especificidade do trabalho em saúde mental, foi deliberada a presença de dois profissionais do serviço na triagem para acolhimento, avaliação conjunta e encaminhamento dos nossos usuários. $\mathrm{O}$ encaminhamento de profissionais do CAPS na triagem seguiu a orientação da SSM que orienta a presença de profissional da enfermagem no acolhimento do serviço para a realização de triagem básica para síndrome gripal e que os atendimentos devem ocupar os espaços externos, evitando a permanência em salas por longos períodos. ${ }^{15}$

Tendo por base oProjeto Institucional do CAPSUERJ e discussão em GT, o serviço foi organizado priorizando a Atenção à Crise - realizada em atendimentos individuais com equipe multidisciplinar para maior suporte - e consultas psiquiátricas para aqueles que necessitam de avaliação de medicação. ${ }^{10}$ Seguindo as indicações de evitar aglomerações para preservar os usuários e equipe, as ferramentas de trabalho convivência, oficinas terapêuticas e atendimentos coletivos - foram suspensas.

A fim de manter a corresponsabilização dos acompanhamentos com os demais serviços do território, foi enviado também um informe aos dispositivos da rede de saúde mental sobre o trabalho de triagem e todas as mudanças que estavam sendo implementadas na PPC e no CAPS UERJ, destacando o quanto isso interferiria no processo de trabalho do serviço.

Durante a primeira semana uma força tarefa avaliou e adiantou as consultas médicas agendadas. Contatos telefônicos foram realizados para antecipar a ida de usuários-familiares ao CAPS, assim como articulações com as Clínicas da Família para dispensação de medicações. Esta conduta garantiu o acesso a medicações e receitas por entendê-las como uma forma de assegurar que os usuários e familiares se sentissem mais protegidos frente à sensação de incerteza produzida pela crise e pela ideia de que o serviço fecharia. Outra importante medida implementada pelo GT foi a listagem e a inclusão de todas as medicações utilizadas pelos nossos usuários que se encontrava em falta na rede de saúde na lista de compra mensal da farmácia da PPC, podendo assim ser utilizada como medicação emergencial.

Prevaleceu-se também o levantamento dos pacientes que fazem uso de medicação injetável de depósito, ao compreendermos que alguns apresentam dificuldades em administrar de forma regular a medicação oral sem o suporte do CAPS. Logo, acompanhar quem faz uso desse tipo de medicação também foi uma frente de trabalho importante para assegurar a manutenção e estratégias de cuidado.

Nesse processo de levantamento dos pacientes supracitados, foram elencados os pacientes com maior grau de desorganização, com rede de cuidado familiar e afetiva frágil, que vivem em condições de vulnerabilidade social extrema, pacientes e/ou familiares incluídos no grupo de risco (idosos, grávidas, comorbidades como câncer, HIV, diabetes, hipertensão, histórico ou com tuberculose) e com outros fatores que representam fragilidade diante do cenário atual. Nesse sentido, foram empreendidos esforços na avaliação de cada um, com o intuito de considerar a possibilidade de alteração da dispensação da medicação prescrita e demandas singulares de cada caso.

Sendo assim, a dispensação medicamentosa foi estruturada de acordo com a organização do sujeito e a existência de rede de suporte, sendo dividida de forma quinzenal, para aqueles com maior dificuldade, ou mensal, para os que possuem maior autonomia e auxilio, conforme avaliação da equipe. Garantiu-se assim, maior segurança no que tange a continuidade do uso da medicação, que contribuiu para uma estabilidade mínima dos casos graves em um momento de crise que, por si só, é desagregador e desorganizador. A dispensação de prescrições mensais ou bimensais foi adotada com o intuito de reduzir a circulação dessas pessoas conforme a pandemia fosse avançando.

Destaca-se, contudo, que o CAPS não preconiza 


\section{Artigo original}

o acompanhamento medicamentoso como única forma de tratamento, deslocando a centralidade desta modalidade para aquelas relacionadas à atenção psicossocial, que foram planejadas e serão explicitadas em momento oportuno neste estudo.

Diminuiu-se a circulação dos usuários no território e no serviço, esperando-se um duplo resultado: a redução da exposição dos usuários a possíveis contaminações e a otimização da força de trabalho no CAPS UERJ que, com um menor quantitativo de frequentadores, pôde atender com mais cuidado os usuários em crise que precisavam de atendimentos presenciais. Aliado a esse trabalho, 70 pacientes foram elegíveis para um monitoramento contínuo nesse período, objetivando a não agudização e/ou manutenção do cuidado dentro dos limites que o panorama atual exige, por entendermos serem casos que demandam acompanhamento intensivo.

Desde o dia 23 de março de 2020, a equipe foi organizada em escalas, na qual cada profissional trabalha em quatro turnos, além das reuniões de acompanhamento semanais virtuais, com auxílio de aplicativos que permitem a videoconferência.

Diariamente, os colaboradores dividiram-se em 2 profissionais para a triagem e os demais dentro da unidade do CAPS UERJ. A comunicação é realizada por meio de dois rádios que facilitaram o atendimento de usuários, a dispensação de receitas, medicações e/ou aplicação de medicação injetável, sem a necessidade da utilização do espaço físico do CAPS. Os profissionais que ficaram dentro do CAPS realizaram acompanhamentos via telefone, bem como atendimentos presenciais, solucionando necessidades de atenção à crise e ofertando suporte aos profissionais que acolhiam usuários na triagem.

Ao estabelecer fluxos de triagem, instalação de estratégias comunitárias para a continuidade do cuidado e encaminhamento dos casos acometidos pela COVID-19 para os setores específicos, notouse que, mesmo não sendo um serviço voltado ao atendimento dos acometidos peloSARS-CoV-2, o CAPS UERJ, empreendeu esforços na direção de assegurar a implementação do que foi preconizado pela OMS, quanto a organização e processo de trabalho que garanta a continuidade dos cuidados em saúde mental.

Produção de cuidados territoriais em saúde mental na conjuntura do distanciamento social

O cuidado em saúde mental demanda singularidades na assistência e manejos dos casos. Dentre as especificidades do público assistido pelo CAPS UERJ, destacou-se a vulnerabilidade para a contaminação e agravamento da COVID-19, relacionada à condição psíquica que pode produzir desorganizações nas atividades diárias, maior exposição a situações de risco, autocuidado prejudicado, alta taxa de comorbidades físicas em consequência do uso prolongado de medicação psiquiátrica, a negligência em relação ao adoecimento físico decorrente de longos períodos de internação em hospitais psiquiátricos e as barreiras de acesso às demais especialidades clínicas para além da saúde mental. No campo psicossocial, apontaramse aquelas relacionadas à situação de pobreza, à convivência familiar e social prejudicada e às condições de moradia.

Seguindo essa lógica, além da articulação do cuidado territorial, a equipe do CAPS apostou, de forma inventiva e responsável, em um acompanhamento intensivo, por meio telefônico. Garante-se assim a continuidade da prestação de cuidados àqueles que deixaram de ir presencialmente ao CAPS, mas que ainda demandam continência. Este trabalho provocou o pensamento e a discussão caso a caso, tendo como principal subsídio a singularidade e a possibilidade do cuidado ofertado pela equipe da unidade.

Os profissionais residentes (serviço social, psicologia, enfermagem) ficaram responsáveis pelas ligações, com capacitação prévia em relação às abordagens, aspectos que mereceram maior atenção, frequência dos contatos e modalidade de comunicação a distância (telefone, aplicativo de mensagens instantâneas, chamadas de vídeo, e-mail). Ressalta-se que estas intervenções não têm a intencionalidade de substituir o espaço físico do CAPS, mas garantir a presença do serviço por meio de instrumentos pouco convencionais na produção de cuidados em saúde mental.

O uso destas tecnologias possibilitou aprofundar o pensamento acerca da organização diária do serviço e o manejo das imprevisibilidades constantes no campo da saúde mental. Neste sentido, ressignificou-se o termo tecnologia ao ir além do simples instrumentoaparelho para a tecnologia de cuidado relacional, uma vez que o contato telefônico passou a marcar a força do vínculo, do papel protagonista do usuário no seu cuidado e do poder que as relações têm para a produção de cuidado em saúde mental. Parte-se do pressuposto que a tecnologia leve é relacional e produzida pelos encontros entre usuário e profissional, anunciando o agir entre os sujeitos implicados com a produção de saúde. ${ }^{16}$ A tecnologia leve ganha relevo quando se 
"opera um jogo de expectativas e produções, criando-se intersubjetivamente alguns momentos interessantes como momentos de falas, escutas e interpretações" ${ }^{\prime 17}$

Nesse caminho inventivo ressignificador das tecnologias, foi criada uma pasta em plataforma de armazenamento on-line contendo 70 documentos individuais para cada usuário acompanhado a distância. Esta pasta foi compartilhada com a equipe de trabalhadores e residentes para que evoluíssem nos documentos o produto das ligações e condutas estabelecidas a partir das demandas do usuário. Destacamos que não se objetivou ligações resolutivas e/ou burocráticas. Os contatos sustentaram o vínculo e, diante da identificação de alguma gravidade, seja o agravamento das questões psíquicas, sejam questões clínicas associadas ou não a sintomas da COVID-19, acionou-se a equipe do dia do CAPS para pensar e articular o cuidado presencial no dispositivo ou com a rede territorial. A instauração destas ferramentas de cuidado objetivou a (re)construção e a (re)significação em prol do estabelecimento de espaços de diálogos, de invenção e de produção de sentidos. ${ }^{18}$

Esse trabalho tem mostrado que faz-se necessário apostar na capacidade inventiva do trabalhador no processo de cuidar, criando linhas de fugas produtoras de forças instituintes, que não estão burocratizadas nos sistemas de saúde. ${ }^{19}$

A cada evolução dos residentes nos documentos individuais dos usuários, foram realizados debates e contribuições pelos preceptores do CAPS UERJ. Este espaço, além de um local de registro das atividades, se apresentou como um dispositivo potente para a discussão coletiva dos casos e possíveis encaminhamentos, reflexões e conexões com o cuidado em saúde mental que rompe com a lógica da institucionalização, apontando para um trabalho vivo que aposta na produção das relações, espaços e objetos de interlocução. ${ }^{20}$

Desta forma, cuidar na perspectiva da atenção psicossocial, rompendo com a lógica manicomial, exige experimentações de novos modos de cuidar do sujeito, por uma disponibilidade de afetar e ser afetado, que pode estar em qualquer sujeito, espaço e saber, desde que se esteja à disposição para o encontro com o diferente. ${ }^{21}$ Deve-se ir mais à frente das redes institucionais construídas e apostar também nas redes vivas que são produzidas no trabalho em acontecimento.

O manejo dos casos via telefone, ainda que apresentando suas limitações, somado à existência presencial de uma equipe multidisciplinar no CAPS, garantiu a atenção aos usuários que entraram em crise, seja pelos impactos do momento atual, seja por outras questões. Importante destacar que, neste cuidado, entendeu-se crise como "um arranjo providencial que se tem à disposição quando todos os recursos psíquicos do sujeito já foram utilizados". 22 Portanto, investir nas necessidades do sujeito em crise e coletar o que pode ser construído a partir desse investimento produz mudanças "em sua qualidade de vida, em sua posição no mundo, em sua liberdade". ${ }^{23}$

Recepcionar e acolher a pessoa em crise remete à noção de tecnologia como correlata de investimento humano que requer intensificação de cuidado. É um processo que depende, não só da estrutura ou de aspectos físicos do acesso, mas dos recursos clínicos da equipe, como atender, escutar, avaliar e discriminar as demandas. ${ }^{24} \mathrm{O}$ acolhimento exige, por um lado, uma ação imediata (urgência) e, por outro, um intervalo de tempo para a resposta (traçar a conduta). Neste intervalo, podemos inscrever processos que envolvem "o tempo do sujeito", mas, também, "o tempo do território", entendendo-o como o lugar da duração, onde as relações se tecem e as dinâmicas sociais são desencadeadas. ${ }^{24}$

\section{Articulações Intra e Inter setoriais no território}

Além das estratégias já discriminadas, destacaramse como intervenções também provocadas pela radicalidade do cuidado territorial, as articulações com serviços da saúde, como a Clínica da Família e demais CAPS, tendo em vista a dificuldade de deslocamento apresentada por alguns usuários. Os CAPS têm valor estratégico na produção de cuidados em saúde mental por meio de ações intersetoriais que fortalecem os laços sociais do usuário em seu território, fortalecimento dos laços familiares e comunitários e, consequentemente, evitando internações em hospitais psiquiátricos. ${ }^{6}$

O resgate da cidadania é um processo pela valorização das pessoas enquanto sujeito de direitos, a fim de fazê-los cidadãos. ${ }^{25}$ Justifica-se a ação em prol da garantia de acesso às medicações e outros cuidados em saúde, articulação com outras instituições e serviços da rede intersetorial, a saber: Centro de Referência da Assistência Social (CRAS), Centro de Referência Especializado da Assistência Social (CREAS), Organizações não Governamentais (ONGs), e com atores do território, como familiares, amigos, vizinhos para suporte e apoio. Destarte, sobressai a importância do conhecimento territorial em suas múltiplas dimensões e a construção de novos territórios 


\section{Artigo original}

de sentido para o sujeito, o que possibilita a produção de cuidado por meio do processo de construção de subjetividades, de identidades, de vínculos, de laços sociais e de pertencimento dos espaços da cidade, garantindo a esses sujeitos a sensação de pertencimento e cidadania.

Após a primeira quinzena de trabalho, os usuários com necessidade de acompanhamento intensivo do CAPS passaram a demandar maior cuidado, apresentaram-se, com mais frequência, presencialmente ou por meio telefônico. Foi possível coletar uma concentração maior de demanda relacionada às questões medicamentosas e de organização do cotidiano da vida. Este fato corroborou um diagnóstico situacional previsto pela equipe: as mudanças nos processos de trabalho, sobretudo com a suspensão das atividades coletivas e de convivência, apresentariam maiores consequências para os usuários a partir da segunda semana de implementação das medidas emergenciais.

A possibilidade de seguir em contato com a equipe e saber que o CAPS seguia em funcionamento caso precisassem de intervenções mais contundentes foi organizador, garantiu maior segurança e sustentou os vínculos com a equipe, conforme recolhido de usuários e familiares.

Diante disso, a atenção à crise e aos trabalhos no território foram apresentando-se como estratégias necessárias, apesar de não preconizadas pelo MS. Devido à agudização de alguns casos foram necessárias visitas domiciliares e institucionais para administrar medicação injetável de depósito e manejo de crises. Ressaltando que a nossa presença no território é convocada em ocorrência do sofrimento psíquico e a necessidade de atendimento conjunto com os demais dispositivos de saúde.

O avanço das contaminações, a prorrogação do tempo de isolamento social e a implementação dos protocolos de atendimentos já relatados nos possibilitou aquecermos redes de suporte e cuidado no território fundamentando as ações no cuidado extramuros. Tal fato produz redes de sociabilidade e de cuidado para além do que é instituído pelo dispositivo, protagonizando os acontecimentos no seu processo de cuidado e construindo diversas conexões na vida, a partir dos encontros. $^{26}$

Logo, tem-se articulado cotidianamente com as demais políticas setoriais, compreendendo o sujeito na sua totalidade e complexidade. Antes da pandemia, o CAPS UERJ ofertava refeições diariamente, para alguns eram as únicas no dia. Com a falta dessa oferta, construiuse uma parceria com o CREAS do território, que passou a fornecer o desjejum para os usuários, viabilizada a partir da doação de parceiros da sociedade civil.

Em conjunto a essa ação, a parceria com o CREAS proporcionou o acesso a informação e a inscrição no Cadastro Único para Programas Sociais para aqueles usuários e familiares que possuem o direito ao auxílio emergencial fornecido pelo Governo Federal pelo período de 3 meses, ${ }^{27}$ bem como, auxiliaram na intensidade do cuidado aos nossos usuários que se encontram em situação de rua e demandam maior suporte da Política de Assistência Social.

Também temos experienciado de forma profícua, e fruto do trabalho anterior à pandemia, a continuidade da composição nos espaços de discussão e articulação da rede intersetorial da AP 2.2, a parceria com ONGs que contribuíram nesse período de forma efetiva com cestas básicas e kits de higiene para os nossos usuários e familiares. Evidenciou-se, assim, o reconhecimento do território como espaço de desenvolvimento de novas interações e de práticas de cuidado em rede.

É fundamental, portanto, pensar a relação dos sujeitos com os territórios para que o trabalho aconteça no CAPS. O território é estratégia central para buscar sentidos e (re)construir as histórias de vida dos sujeitos, por meio da incorporação de dispositivos territorializados que são utilizados no cotidiano. As ações territoriais produzidas pelo serviço tem sido um dispositivo que oportuniza a ressignificação dos espaços da cidade, construção de laços sociais, convívio social, abertura de diálogo e reinvenção da vida no seu cotidiano.

\section{Considerações finais}

Por maiscontingentequeseja a vida esuas expressões, incluindo suas dores, e por mais aterrorizadoras que sejam as condições de crises (por todos aqueles que as vivem - usuários, familiares e profissionais), apostamos que apenas relações com vínculos sólidos e estratégias de cuidado consistentes, seguras e corajosas são capazes de conter acontecimentos que indicam uma situação de desordem, de desestruturação e de caos.

Reinventar as estratégias em prol da continuidade da radicalização do cuidado territorial e da utilização de tecnologias leves de produção de saúde em situação de crise é promover um intenso investimento humano, ou seja, assumir o lugar de promotor de efetivas mudanças visando o sujeito.

Em uma avaliação inicial, a pandemia e seus 
desdobramentos no cenário socioeconômico e cultural, e mais diretamente nas trajetórias dos nossos usuários e seus familiares, ressaltou a necessidade do trabalho no território, estreitando a comunicação com outros serviços de saúde e de outras políticas públicas. Também ficou evidente, a partir dos relatos de usuários e familiares, a potência da convivência física entre os profissionais e usuários e também entre os próprios usuários no cotidiano do CAPS, e como essa convivência aplaca o sofrimento, possibilita a construção singular e coletiva das diversas possibilidades de viver e reforça o lugar do CAPS como uma referência de cuidado e acolhimento para usuários e familiares.

\section{Referências}

1. World Health Organization. Access the guidance COVID-19 Partners Platform based on Operational Planning Guidance Training modules: Operational Planning Guidelines and COVID-19 Partners Platform. 2020 [acesso em 07 abr 2020]. Disponível em: https://openwho.org/courses/UNCT-COVID19preparedness-and-response-EN.

2. Worldometer. Coronavirus Update (Live): $1,266,782$ Cases and 69,177 Deaths from COVID-19 Virus Outbreak - Worldometer. Worldometer. 2020 [acesso em 09 abr 2020]. Disponível em: https://www.worldometers.info/coronavirus/?.

3. World Health Organization. COVID-19: operational guidance for maintaining essential health services during an outbreak: interim guidance, 25 March 2020. 2020 [acesso em 09 abr 2020 ]. Disponível em: https://apps.who.int/iris/handle/10665/331561.

4. World Health Organization. Director-General's opening remarks at the media briefing on COVID-19 - 30 March 2020. 2020 [acesso em 10 abr 2020]. Disponivel em: https://www.who.int/ $\mathrm{dg} / \mathrm{speeches/detail/who-director-general-s-opening-remarksat-}$ the-media-briefing-on-covid-19---30-march-2020.

5. Brasil. Ministério da Saúde. Plano de Contingência Nacional para Infecção Humana pelo novo Coronavírus - COVID-19. Brasília: Centro de Operações de Emergências de Saúde Pública (COE-COVID-19) .Brasília, DF: Diário Oficial [da] República Federativa do Brasil. 2020. Disponível em: https://portalarquivos2.saude.gov.br/images/pdf/2020/fevereiro/13/plano-contingencia-coronavirus-COVID19.pdf). Acesso em: 08 de abr. 2020.

6. Rafael RMR, Neto M, Carvalho MMB, et al. Epidemiologia, políticas públicas e COVID-19. Rev enferm UERJ. 2020; 28. Capa.

7. Minayo MCS. Pesquisa Social: teoria, método e criatividade. $34^{a}$ ed. Petrópolis: Vozes; 2014. p. 67-79.

8. Egry EY. Saúde coletiva: construindo um novo método em enfermagem. 1 ed. São Paulo: Ícone; 1996.

9. Brasil. IBGE. Censo demográfico 2010. IBGE: Instituto Brasileiro de Geografia). 2010.

10. Barbosa AS, East AL, Chaves RCS, et al. Centro de atenção psicossocial da universidade do estado do rio de janeiro: caps uerj - cuidado, extensão, ensino e pesquisa. 2 ed. Rio de Janeiro, RJ: Projeto Institucional. 2019.

11. Brasil. Ministério da Saúde. Portaria $n^{\circ} 3.088$, de 23 de dezembro de 2011. Institui a Rede de Atenção Psicossocial para pessoas com sofrimento ou transtorno mental e com necessidades decorrentes do uso de crack, álcool e outras drogas, no âmbito do Sistema Único de Saúde (SUS). Brasília (DF): Ministério da Saúde; 2011

12. Lima TCS, Mioto RCT, Dal Prá KR. A documentação no cotidiano da intervenção dos assistentes sociais: algumas considerações acerca do diário de campo. Textos \& Contextos. 2007;6(1):93-104.

13. Brasil. Ministério da Saúde. Conselho Nacional de Saúde. Resolução 510/2016 - Normas para pesquisa envolvendo seres humanos. 2 ed. Brasília, DF: Diário Oficial [da] República Federativa do Brasil.2016.

14. World Health Organization. Severe Acute Respiratory Infections Treatment Centre. World Health Organization. [Internet]. 2020 [acesso em 07 abr 2020]. Disponível em: https://www.who.int/ publications-detail/severe-acute-respiratory-infections-treatment-centre).

15. Prefeitura do município do Rio de Janeiro. Secretaria Municipal de Saúde. Resolução n. 4333, de 8 de março de 2020. Recomendações para os CAPS do município do Rio de Janeiro referentes a COVID-19. Diário Oficial [do] Município do Rio de Janeiro. 2020 mar 18. p.10-11.

16. Merhy E. $\mathrm{O}$ ato de governar as tensões constitutivas do agir em saúde como desafio permanente de algumas estratégias gerenciais. Cienc. Saude Colet. 1999;4(2):305-314

17. Merhy, E. $O$ ato de governar as tensões constitutivas do agir em saúde como desafio permanente de algumas estratégias gerenciais. Cienc. Saude Colet. 1999;4(2):318.

18. Silva LR. Vivendo em liberdade: desinstitucionalização em um Centro de Atenção Psicossocial [monografia]. Rio de Janeiro: Universidade do Estado do Rio de Janeiro (UERJ), 2019.

19. Franco TB. As redes na micropolítica do processo de trabalho em saúde. In: Pinheiros R, Mattos RA. Gestão em redes: práticas de avaliação, formação e participação na saúde. Rio de Janeiro: Abrasco, 2006. p. 459-473

20. Rotelli F. A instituição inventada. In: Nicácio MF. Desinstitucionalização. 2 ed. São Paulo, Hucitec, 2001

21. Romagnolli RC, Paulon SM, Amorim AKMA, et al. Por uma clínica da resistência: experimentações desinstitucionalizantes em tempos de biopolítica. Interface (Botucatu).2009;13(30):199-207.

22. Vidal FB, Braga ACS, Sodré AROM. A abordagem da crise na psicose. In: Silva MVO. IN-tensa/EX-tensa: a clínica psicossocial das psicoses. Salvador: LEV - Laboratório de Estudos Vinculares/UFBA, 2007. p.151-68.

23. Oliveira M, Mota, E. Entrevista com Eduardo Mota e Marcus Vinícius Oliveira, supervisores do Programa de Intensificação de Cuidados a Pacientes Psicóticos. In: Silva MVO. IN-tensa/ EX-tensa: a clínica psicossocial das psicoses. Salvador: LEV Laboratório de Estudos Vinculares/UFBA, 2007. p.15-36.

24. Schmidt M, Figueiredo A. Acesso, acolhimento e acompanhamento: três desafios para o cotidiano da clínica em saúde mental. Rev. Latinoam. Psicopat. Fund.2009; 12(1):130-40.

25. Leal E, Delgado PGG. Clínica e cotidiano: o CAPS como dispositivo de desinstitucionalização. In: Pinheiro R, Gulijor AP, Junior AGDS, Mattos RA. . Desinstitucionalização na saúde mental: contribuições para estudos avaliativos. 1 ed. Rio de Janeiro: Cepesc; IMS/LAPPIS; Abrasco, 2007. p.137-54.

26. Belini MG, Hirdes A. Projeto Morada São Pedro: da institucionalização à desinstitucionalização em saúde mental. Texto Contexto Enferm. 2006; 15(4): 562-569.

27. Bertussi D. et al. Viagem cartográfica: pelos trilhos e desvios. In: Mattos RA, Baptista TWF. Caminhos para análise das políticas de saúde. 1 ed. Porto Alegre, Rede Unida, 2011. p. 306-324.

28. Brasil. Decreto-lei n¹066, de 30 de Março de 2020. Diário Oficial [da] República Federativa do Brasil, Poder Executivo, Brasília, DF, 02 de abr. de 2020, seção I, p.1-2. 